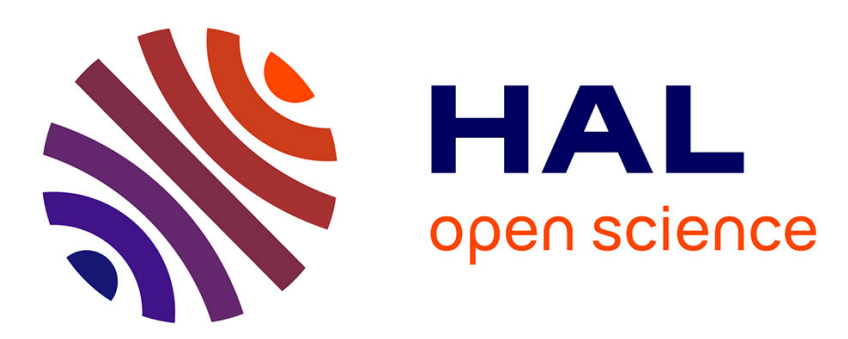

\title{
Attosecond imaging of XUV-induced atomic photoemission and Auger decay in strong laser fields
}

S Zherebtsov, A Wirth, T Uphues, I Znakovskaya, O Herrwerth, J Gagnon, M Korbman, V S Yakovlev, M J J Vrakking, M Drescher, et al.

\section{- To cite this version:}

S Zherebtsov, A Wirth, T Uphues, I Znakovskaya, O Herrwerth, et al.. Attosecond imaging of XUVinduced atomic photoemission and Auger decay in strong laser fields. Journal of Physics B: Atomic, Molecular and Optical Physics, 2011, 44 (10), pp.105601. 10.1088/0953-4075/44/10/105601 . hal00617383

\section{HAL Id: hal-00617383 \\ https://hal.science/hal-00617383}

Submitted on 28 Aug 2011

HAL is a multi-disciplinary open access archive for the deposit and dissemination of scientific research documents, whether they are published or not. The documents may come from teaching and research institutions in France or abroad, or from public or private research centers.
L'archive ouverte pluridisciplinaire HAL, est destinée au dépôt et à la diffusion de documents scientifiques de niveau recherche, publiés ou non, émanant des établissements d'enseignement et de recherche français ou étrangers, des laboratoires publics ou privés. 


\title{
Attosecond imaging of XUV-induced atomic photoemission and Auger decay in strong laser fields
}

\author{
S. Zherebtsov ${ }^{1}$, A. Wirth ${ }^{1}$, T. Uphues ${ }^{1}$, I. Znakovskaya ${ }^{1}$, O. Herrwerth ${ }^{1}$, J. Gagnon ${ }^{1}$, M. \\ Korbman $^{1}$, V.S. Yakovlev ${ }^{1,2}$, M.J.J. Vrakking ${ }^{3,4}$, M. Drescher ${ }^{5}$, M.F. Kling ${ }^{1,6, *}$ \\ ${ }^{1}$ Max-Planck Institut für Quantenoptik, Hans-Kopfermann-Str. 1, D-85748 Garching, \\ Germany \\ ${ }^{2}$ Fakultät für Physik, Ludwig-Maximilians Universität, Am Coulombwall 1, 85748 \\ Garching, Germany \\ ${ }^{3}$ FOM Institute AMOLF, Science Park 104, 1098 XG Amsterdam, The Netherlands \\ ${ }^{4}$ Max-Born-Institut, Max-Born Strasse 2A, D-12489 Berlin, Germany \\ ${ }^{5}$ Fachbereich für Physik, Universität Hamburg, Luruper Chausee 149, D-22761 \\ Hamburg, Germany \\ ${ }^{6}$ J. R. Macdonald Laboratory, Department of Physics, Kansas State University, \\ Manhattan, KS 66506, USA \\ *E-mail: matthias.kling@mpq.mpg.de
}

\begin{abstract}
Velocity-map imaging has been employed to study the photoemission in $\mathrm{Ne}$ and $\mathrm{N}_{4,5} \mathrm{OO}$ Auger decay in Xe induced by an isolated $85 \mathrm{eV}$ extreme ultraviolet (XUV) pulse in the presence of a strong few-cycle near-infrared (NIR) laser field. Full three dimensional momentum information about the released electrons was obtained. The NIR and XUV pulse parameters were extracted from the measured Ne streaking traces using a FROG CRAB retrieval algorithm. The attosecond measurements of the Auger decay in Xe show pronounced broadening of the Auger lines corresponding to the formation of sidebands. The temporal evolution of the sideband signals and their asymmetry along the laser polarization axis exhibit oscillations similar to those known from attosecond streaking measurements. The experimental results are in good agreement with model calculations based on an analytical solution of the Schrödinger equation within the strong field approximation.
\end{abstract}

\section{Introduction}

Along with the development of ultrashort laser pulses, significant advances in physics, chemistry and biology were achieved. The elementary motions of atoms in molecules occur on femtosecond time scales [1]. The development of attosecond (1 as $=10^{-18} \mathrm{~s}$ ) metrology has made real-time studies of electron dynamics possible [2, 3]. Excitation of atoms by XUV radiation can lead to the ejection of deeply bound (core) electrons. The created vacancies correspond to highly unstable states that may undergo multi-electron relaxation dynamics as they decay. The decay may take place in a single step, but usually occurs as a cascade of successive radiative or non-radiative processes via transient intermediate states. Atomic inner-shell relaxation therefore represents a showcase example of complex multi-particle dynamics. While the final steps in a relaxation 
cascade, which involve the emission of a photon, may extend to pico-, nano-, or even microsecond durations [4], the early steps often involve radiationless Auger decay which may take place within a few femtoseconds [5]. In pioneering work [5] the femtosecond Auger decay following attosecond excitation of $\mathrm{Kr}$ was measured with few-cycle pulses without CEP stabilization. Cascaded Auger decay in $\mathrm{Xe} \mathrm{[6]} \mathrm{and} \mathrm{Kr}$ [7] were studied by ionization chronoscopy, where the relaxation times of the Auger process were obtained from the time-dependent ion yields. Quantum mechanical modelling of time-resolved Auger emission predicts a transition from the streaking regime to the formation of sidebands for Auger decay times comparable to the period of the laser field [8]. Recent theoretical calculations for the laser assisted KLL Auger transitions in Ne predict sideband structure different from the conventional equidistant sidebands. The position and shape of the energetic Auger emission exhibit a strong dependence on both the time delay between the XUV and NIR pulse [9] and the emission angle [10].

We carried out XUV pump / NIR probe experiments utilizing velocity-map imaging to retrieve the full three dimensional momentum distribution of electrons emitted after attosecond XUV excitation of $\mathrm{Ne}$ and $\mathrm{Xe}$ at $85 \mathrm{eV}$ in a strong NIR laser field. Phasestabilized few-cycle $(\sim 4 \mathrm{fs})$ NIR pulses at $740 \mathrm{~nm}$ carrier wavelength and intensities that were varied between $2 \times 10^{12}$ and $2 \times 10^{13} \mathrm{~W} / \mathrm{cm}^{2}$ were employed in the attosecond streaking experiments. Streaking of electrons emitted from the $2 p$ photoemission line in $\mathrm{Ne}$ with the few-cycle laser field was observed. The time-resolved Auger emission in Xe exhibits pronounced sub-cycle variations in the sideband structure, characteristic for the intermediate regime, when the core-hole relaxation time is on the order of the period of the laser field as predicted by previous theoretical work [8]. The results presented here show the potential of the combination of state-of-the-art attosecond metrology with 3D imaging.

\section{Experiment}

\subsection{Experimental setup}

The output of an amplified, phase-stabilized $3 \mathrm{kHz}$ Ti:sapphire laser system (Femtolasers, Femtopower Compact Pro) with $900 \mu \mathrm{J}$ pulse energy and sub-25 fs pulse duration was spectrally broadened in a Ne-filled hollow-core fiber and compressed by a combination of chirped mirrors to a pulse duration of about 4 fs [11]. These ultrashort laser pulses were focused into a Ne gas target where XUV harmonics with energies up to $\sim 100 \mathrm{eV}$ were produced. The collinearly propagating XUV and laser beams passed through a 200 $\mathrm{nm}$ thick, $3 \mathrm{~mm}$ diameter $\mathrm{Zr}$ foil placed on a $5 \mu \mathrm{m}$ thick nitrocellulose pellicle. Behind the filter the annular NIR beam and the XUV beam in the center were focused into the target gas jet with a two-component broadband $\mathrm{Mo} / \mathrm{Si}$ multilayer mirror with a focal length of $125 \mathrm{~mm}$. The XUV beam is focussed to a diameter of ca. $2 \mu \mathrm{m}$, while the NIR beam is focussed to ca. $30 \mu \mathrm{m}$ in diameter. This mirror and the $\mathrm{Zr}$ foil together act as a bandpass filter for the harmonic spectrum that spectrally isolates a single attosecond pulse from the cut-off region. The inner mirror was placed on an ultra high precision piezo translation stage to control the relative delay between the attosecond pulses and the few-cycle NIR field. The intensity of the NIR field in the focus was adjusted with a 
variable iris which was placed in front of the pellicle. The schematic of the attosecond velocity map imaging part of the setup is illustrated in figure 1. Electrons, generated at the crossing of the laser beam and an effusive gas jet, formed with a capillary integrated in the repeller electrode of the VMI spectrometer [12], were focused by the electron optics of a VMI spectrometer onto a microchannel plate (MCP)/phosphor screen assembly. The image of the projection of the electron momentum distribution onto the detector plane was recorded with a Peltier-cooled CCD camera. The polarization of the NIR and the XUV pulses were chosen to be in the plane of the detector ( $x-y$ plane). To obtain the electron three dimentional momentum distribution from the recorded images an iterative inversion procedure was used [13].

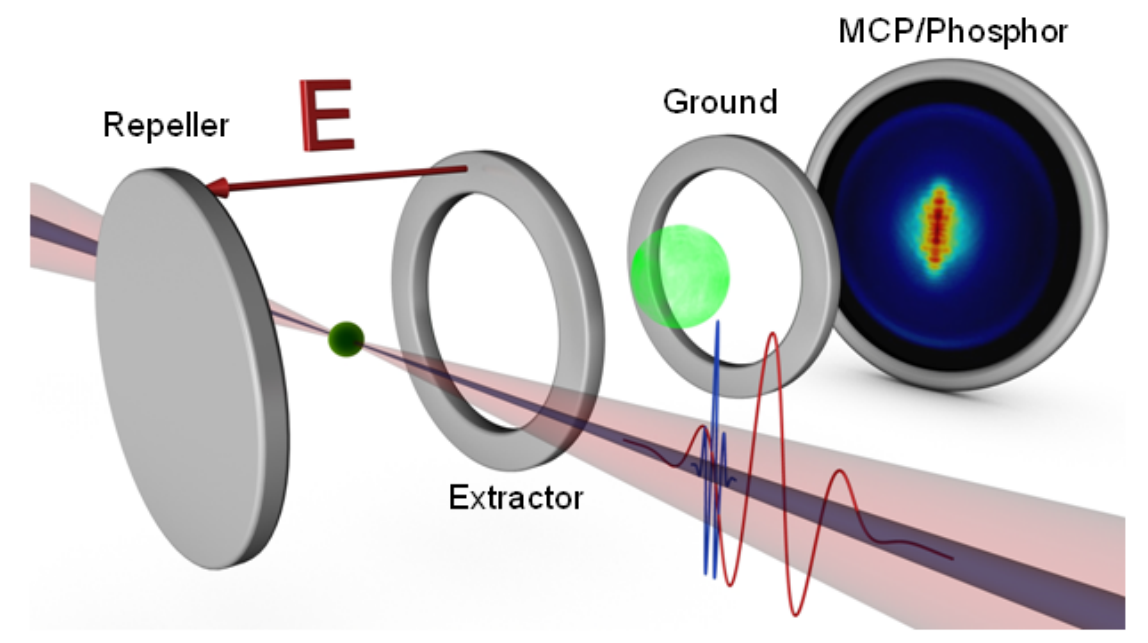

Figure 1. Schematic of attosecond velocity-map imaging. Few-cycle NIR and XUV light fields have polarizations as indicated in the figure. The emitted electrons (green spheres) are focused by the electron optics (repeller, extractor, and ground electrodes) onto an MCP-phosphor screen assembly, where an image of the projection of the electron momentum distribution is formed.

\subsection{Attosecond imaging of electron photoemission in Ne}

The interaction of $\mathrm{Ne}$ atoms with the XUV pulses (centered at $85 \mathrm{eV}$, FWHM of $8 \mathrm{eV}$ ) can lead to photoemission of electrons from the $2 \mathrm{~s}$ and $2 \mathrm{p}$ shells. Figure 2 shows a momentum map (a) and kinetic energy spectrum (b) of the photoelectrons measured at a relative delay of $0.4 \mathrm{fs}$ with the overlap between the XUV and NIR pulses being at $5.8 \mathrm{fs}$. At the relative delay chosen for Figure 2(a) the XUV and NIR pulses do not overlap and the NIR pulse precedes the XUV pulse. The image on the camera was integrated for $\sim 1$ min. The signal in the center of the momentum map stems from above threshold ionization (ATI) of Ne in the strong NIR field, while the outer ring is the contribution from the XUV photo-emitted $2 p$ electrons. The signal from the photoemitted $2 \mathrm{~s}$ electrons overlaps with high energy ATI electrons, located in the center of the image (as indicated in Fig. 2). The amplitude of the streaking curve (see below) suggests that the peak intensity of the few-cycle laser field was about $1 \cdot 10^{13} \mathrm{~W} / \mathrm{cm}^{2}$. It should be noted that a significantly higher value of the laser intensity $\left(4 \cdot 10^{13} \mathrm{~W} / \mathrm{cm}^{2}\right)$ is determined from the cut off in the ATI contribution. This discrepancy might be due to a non-perfect spatial 
overlap of the foci of the XUV and NIR beams. The streaking waveform of the $2 p$ electrons, however, was not affected by the ATI electrons.
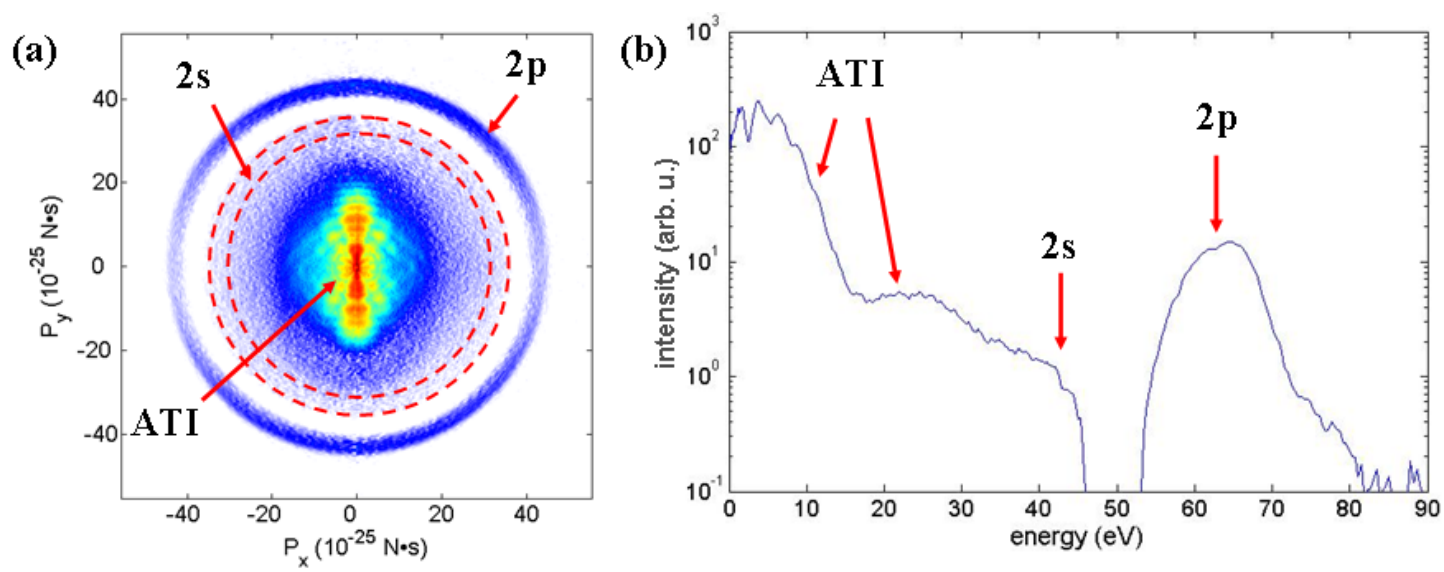

Figure 2. (a) Momentum map (log color scale) measured in the two-color (XUV and NIR) ionization of $\mathrm{Ne}$ at a delay of 0.4 fs (indicated by a white arrow in Fig. 4 (a)). The polarization of the electric field was along the $p_{\mathrm{y}}$ axis. (b) Spectrum obtained from the momentum image by integration over an angular range of 40 degrees along the polarization vector in the positive $p_{\mathrm{y}}$ direction.

If the exciting XUV pulse is short and the electron wavepacket leaves the atom within a small fraction of the laser wave cycle the momentum of the freed electron is changed along the laser vector potential:

$$
\vec{p}_{f}(t)=\vec{p}_{0}-e \int_{t}^{\infty} \vec{E}_{L}\left(t^{\prime}\right) d t^{\prime}=\vec{p}_{0}-e \vec{A}(t) \text { (for } e>0 \text { ), }
$$

where $\vec{p}_{f}(t)$ is the final momentum of the photoelectron emitted in the presence of the laser field, $p_{0}=\sqrt{2 m\left(\hbar \omega_{X U V}-W_{\text {bind }}\right)}$ is the momentum of the photoelectron in the absence of the laser field, and $\vec{A}(t)$ is the vector potential of the laser field at the instance of electron emission [14]. Momentum maps measured at three different delay settings are shown in figure 3 . The momentum shift of the XUV photo-emitted $2 p$ electrons along the laser polarization direction is weak but can be seen by comparing it to the unshifted red circle that is centered around the ATI signal, which appears in the center of the images and stems predominantly from the creation of $\mathrm{Ne}^{+}$. The angle-resolved streaking behaviour is qualitatively similar to data that was obtained by Mauritsson et al. [15] in Ar with a train of attosecond pulses spaced by a full wave cycle. The use of multiple attosecond pulses, however, results in an interference that leads to multiple rings around the central emission energy. This interference is absent in the experimental data presented here, where isolated attosecond pulses were used. The angle-resolved streaking of photoelectrons produced by attosecond XUV pulses in an intense NIR laser field was discussed in earlier theoretical work (see e.g. $[9,16,17]$ ). Due to the different parameters used in these theoretical studies, a quantitative comparison was not attempted here. 
(a)

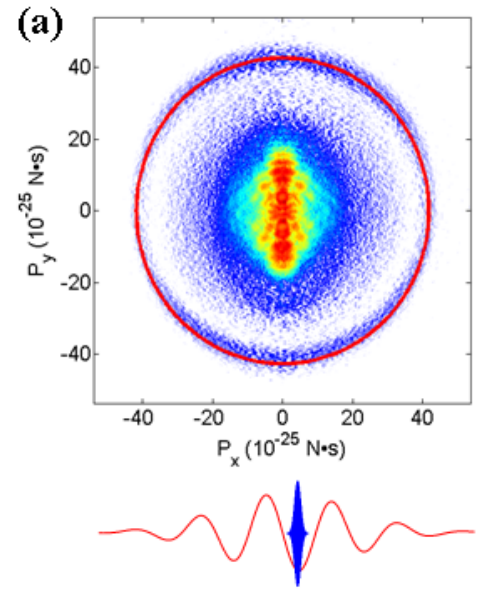

(b)
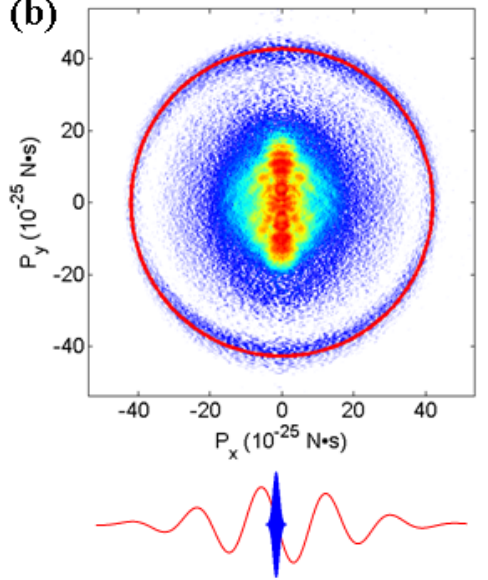

(c)

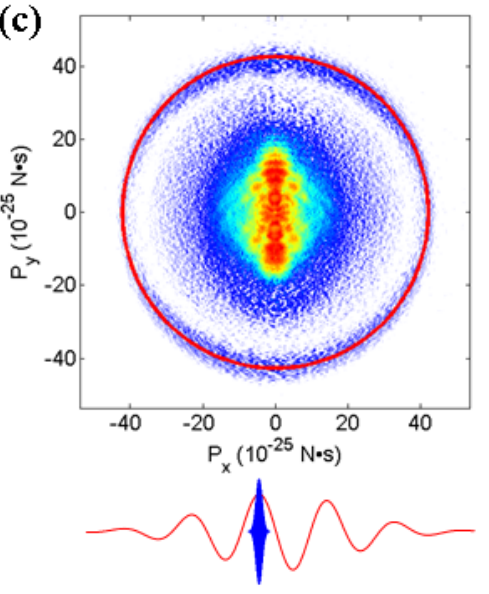

Figure 3. Momentum maps of photoelectrons measured in $\mathrm{Ne}$ at three different delays between the XUV and NIR pulses as depicted below the images: at the minimum (a), zero (b) and maximum (c) of the vector potential (red waveform). To make the shift of the $2 p$ photoemission line by the NIR field along the polarization axis more discernible, a red circle is displayed in each image, which is centered around zero $p_{\mathrm{x}}$ and $p_{\mathrm{y}}$ momenta.

Figure 4(a) shows the $2 p$ photoemission region of the spectrogram obtained by angular integration of the electron momentum maps within a 40 degree angular range in the positive $p_{\mathrm{y}}$ direction. The streaking of the photoelectrons by the few-cycle laser field is clearly visible. To extract information on the XUV pulse, a FROG CRAB retrieval algorithm was employed [18]. It was shown in [19] that a collection angle as large as 40 degrees has no detrimental effect on the retrieval. Figure 4(b) shows the retrieved spectrogram, and figure 4(c) shows the extracted temporal intensity profile and phase of the XUV pulse. The extracted pulse duration of 230 as is close to the Fourier limit for the XUV pulse (bandwidth of $\sim 8 \mathrm{eV}$ ). The retrieval shows only a small variation of the phase across the pulse. 
(a)

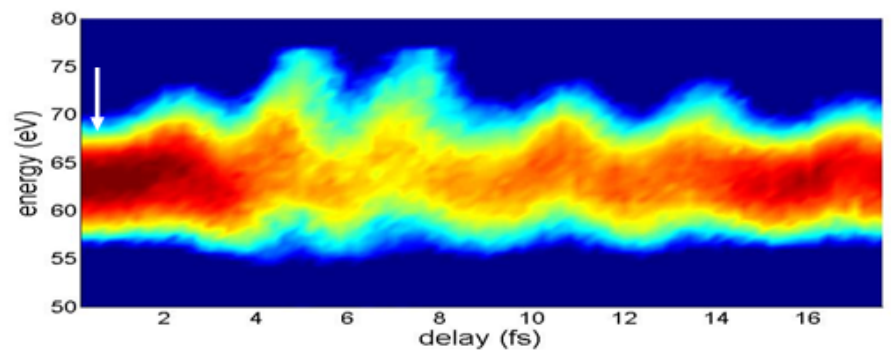

(b)

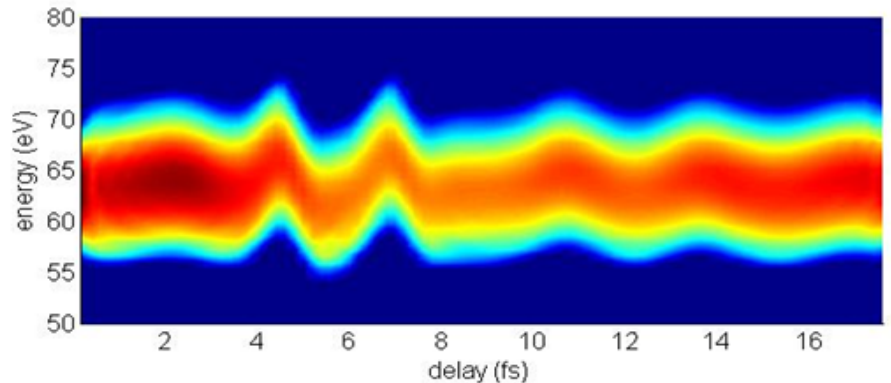

(c)

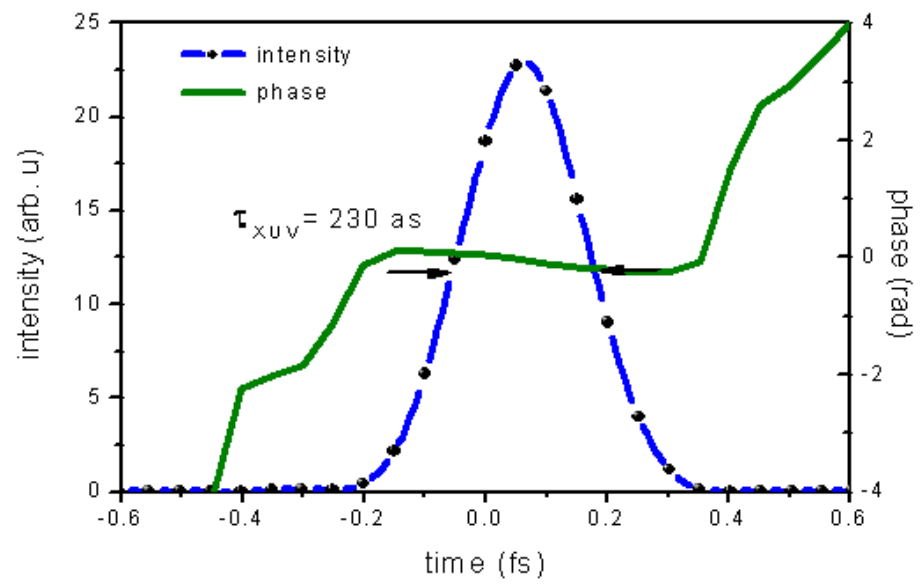

Figure 4. Measured attosecond streaking spectrogram (a), and reconstructed spectrogram after 1000 iterations of the FROG CRAB algorithm (b). (c) Retrieved temporal intensity profile and phase of the attosecond XUV pulse.

\subsection{Tracing Auger decay in Xe with attosecond streaking}

We also studied the XUV-induced electron emission from Xe atoms by attosecond streaking. The XUV radiation (with parameters determined in the $\mathrm{Ne}$ streaking experiments) mostly ionizes from the $4 \mathrm{~d}$ shell creating a $\mathrm{Xe}^{+}$ion with an inner shell vacancy [20]. Subsequently, this excitation predominantly decays via an Auger process to $\mathrm{Xe}^{2+}$ in a $5 \mathrm{p}^{-2}$ configuration [21].

A typical electron momentum map measured in Xe after XUV excitation in the presence of a weak NIR field is shown in figure 5(a). To reduce the ATI contribution in the center of the image, a rather low NIR intensity $\left(1 \cdot 10^{12} \mathrm{~W} / \mathrm{cm}^{2}\right)$, which was determined from the streaking of the $4 \mathrm{~d}$ electrons as described below, was used in the experiment. 
The outermost broad ring stems from the XUV photoemission of $5 \mathrm{~s}$ and $5 \mathrm{p}$ electrons, which show preferential emission along the polarization direction. The bright isotropic ring just inside the dashed red circle in figure 5(a) is due to $\mathrm{Xe} \mathrm{N}_{4,5} \mathrm{OO}$ Auger electrons [22]. The next contribution towards smaller radii corresponds to the emission of $4 \mathrm{~d}$ electrons and the center of the image is dominated by ATI from the interaction of Xe with the NIR field. The spectrogram in figure 5(b) shows time-dependent electron kinetic energy spectra for the Auger and $4 \mathrm{~d}$ emission region that were obtained by integration of the momentum maps within 40 degrees along the positive $p_{\mathrm{y}}$ direction. A similar spectrogram was obtained in the opposite direction of the laser polarization. Significant broadening of the Auger lines at delays of several femtoseconds after the maximum energy shift of the streaked $4 \mathrm{~d}$ electrons (marked by the black arrow) can be seen (dashed red box). For the analysis of the Auger electron dynamics we used the following Auger lines (line numbering follows ref. [23]): line 3 (initial vacancy N5, final state $5 p^{4}:{ }^{3} \mathrm{P}_{0}$, Auger energy $34.21 \mathrm{eV}$ ) and line 4 (initial vacancy N4, final state $5 \mathrm{p}^{4}:{ }^{1} \mathrm{D}_{2}$, Auger energy $34.07 \mathrm{eV})$. Due to the limited resolution of our detector $(\sim 1 \mathrm{eV}$ at $34 \mathrm{eV})$ these two lines appear as a single peak in the spectrum (indicated by a dashed red circle in figure 5 (a) and a red arrow in figures 5 (b) and (c)). A more detailed analysis of the temporal evolution of these Auger lines is presented in the following section. We focus in this discussion on the angle-integrated Auger electron emission towards the two directions along the laser polarization axis. Along this axis a significant directional asymmetry is discernible.
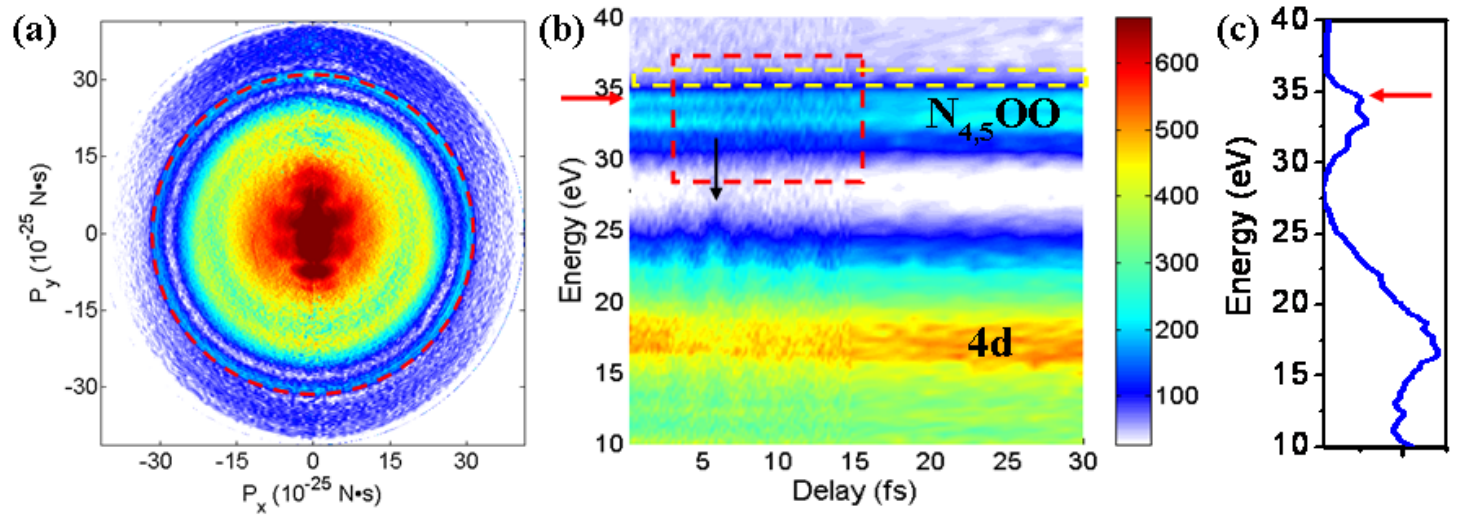

Figure 5. (a) Momentum map of photoelectrons measured in the two-color ionization of $\mathrm{Xe}$ at a delay of $27 \mathrm{fs}$. (b) Map of electron kinetic energy spectra after attosecond excitation of $\mathrm{Xe}$ as a function of the delay time between the $85 \mathrm{eV}$ pump and a phasestable few-cycle NIR probe pulse. (c) Photoelectron spectrum measured at a delay where the XUV pulse precedes the NIR pulse and there is no temporal overlap between the XUV and NIR pulses. The black arrow in panel (b) indicates the maximum energy shift of the streaked $4 \mathrm{~d}$ electrons.

\section{Modeling of the streaking of the Xe Auger decay}

Our model calculations are based on an analytical solution of the Schrödinger equation within the strong field approximation $[5,8]$. The equation for the delay dependent electron spectrum has the form (in atomic units): 
$S(p, \tau)=|b(p)|^{2} \propto\left|\int_{\infty}^{\infty} e^{-\frac{i}{2} \int_{t^{\prime}}^{\infty}\left(p+A\left(t^{\prime \prime}\right)\right)^{2} d t^{\prime \prime}+i W_{k i n} t^{\prime}} \sqrt{\rho\left(t^{\prime}+\tau\right)} d t^{\prime}\right|^{2}$

where $b(p)$ is the positive-energy part of the momentum space wave function of the electron after passage of both the XUV and the few-cycle laser pulses, $A(t)$ is the vector potential of the laser pulse, $W_{k i n}$ is the kinetic energy of the Auger electrons in the absence of the laser field, $\rho(t)$ is the population of the Auger state, and $\tau$ is the time delay between the XUV and the NIR pulses.

Since the XUV pulse duration is much shorter than the lifetime of the generated core vacancy, the temporal evolution of the Auger state population in the absence of the external laser field can be approximated by:

$\rho(t) \propto \int_{-\infty}^{t} e^{-\Gamma\left(t-t^{\prime}\right)} I_{X U V}\left(t^{\prime}\right) d t^{\prime}$,

where $I_{X U V}$ is the intensity profile of the XUV pulse, and $\Gamma$ is the Auger decay rate.

In order to obtain information about the vector potential of the NIR pulse, the mean energy of the $4 \mathrm{~d}$ photoemission line was calculated for each delay point. Note that the NIR vector potentials in the $\mathrm{Ne}$ and Xe experiments were different, so that a retrieval of the NIR vector potential from the Xe data was necessary in the analysis. In the Auger calculation we used the XUV pulse duration that was extracted with the FROG CRAB retrieval algorithm from the streaking measurements in $\mathrm{Ne}$ at similar experimental conditions (pulse duration $\sim 230$ as). Note that even though the streaking pulses were different in both measurements, the XUV pulses were the same and the retrieval for the $\mathrm{Ne}$ measurement was more reliable. Figure 6(a) shows a simulated electron kinetic energy map for the overlapping Auger lines 3 and 4 following the nomenclature of ref. [23], as was discussed in the previous section. Interaction of the emitted Auger electrons with the laser field leads to the appearance of sidebands spaced by multiples of the NIR photon energy from the energy of the Auger lines. The sidebands show a pronounced temporal substructure resembling the NIR field oscillations, indicating that the Auger relaxation happens on a time scale comparable to the cycle period of the laser field.

To compare the calculations with the experimental results, the spectrograms were integrated in the energy interval of the upper sideband (dashed yellow box in figures 5(b) and 6(a)). The simulated signal also includes a weak, incoherently added contribution from the second order sideband of the Auger lines 7 and 8 (line numbering follows [23]) around $32 \mathrm{eV}$ (not shown in figure 6(a)). In the calculations Auger decay times derived from synchrotron measurements for the $4 \mathrm{~d}_{3 / 2}$ and $4 \mathrm{~d}_{5 / 2}$ holes $(6.3 \pm 0.2 \mathrm{fs}$ and $5.9 \pm 0.2 \mathrm{fs}$, respectively) were used [24]. The results of the simulation and the experimental data are shown together in figure 6(b). Note that a complete FROG CRAB analysis of the experimental data was not yet possible due to the limited count rate in these first angleresolved measurements. The sub-cycle variation in the number of electrons scattered into the sidebands by the NIR field are present both in the simulation and experimental data. These variations are exactly out of phase for the upper and lower direction. While smaller deviations between the simulated curves for the upper and lower emission direction can be found within the rise of the sideband signal, its decay is well represented by the 
simulations. The deviations between the experimental data and the simulation probably originate from the uncertainties in the determination of the vector potential.

(a)

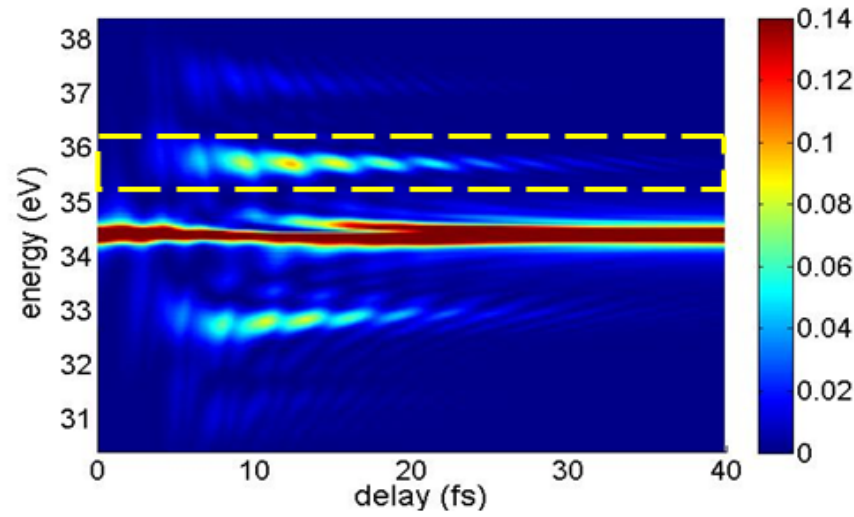

(b)
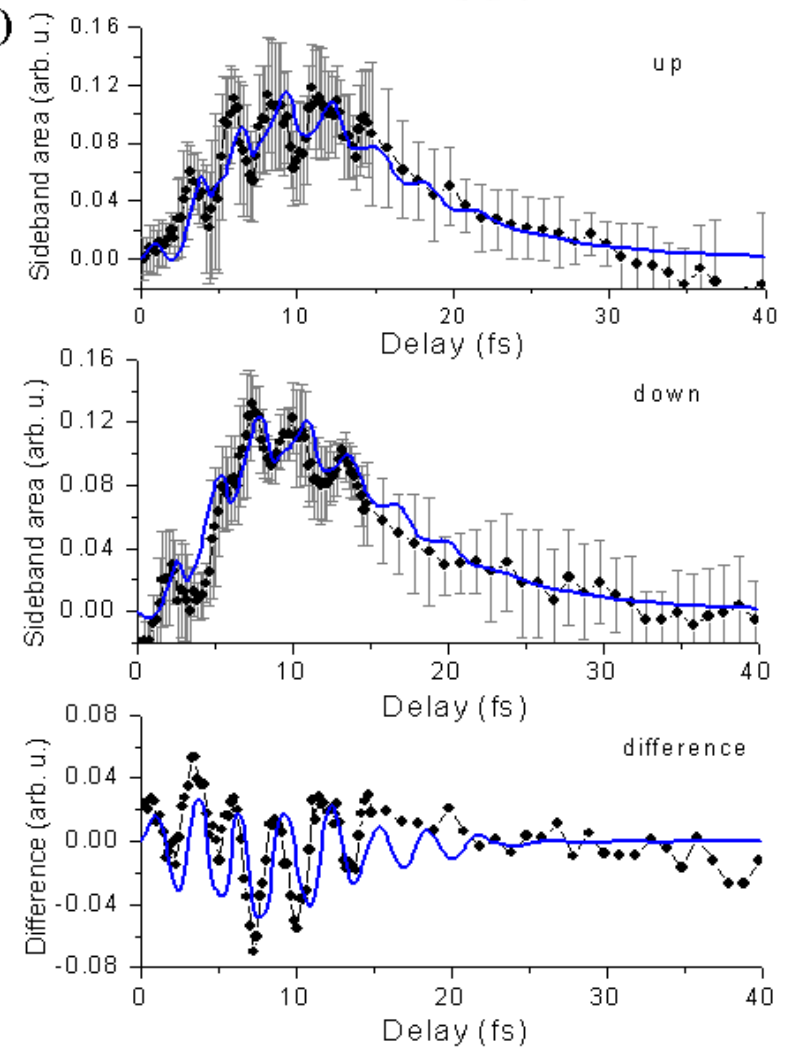

Figure 6. (a) Simulated time dependent spectra of a single Auger line in the region of interest. The interaction with the NIR streaking field leads to the generation of sidebands, which in this case show the oscillatory substructure of the NIR field. (b) Comparison of integrated model calculations (blue) for the upper first sideband (dashed yellow box) in the two emission directions with the experimental data (black). The experimental data were temporally averaged over 5 individual data points (lowering the temporal resolution but improving the signal-to-noise of the experimental data).

\section{Conclusions and Outlook}


Photoemission and Auger decay in strong few-cycle laser fields were studied after the photoexcitation of $\mathrm{Ne}$ and Xe by isolated attosecond XUV pulses using the velocity map imaging technique. Attosecond streaking of the $2 p$ photoelectrons from $\mathrm{Ne}$ allowed us to recover the XUV and NIR pulses using the FROG CRAB algorithm. Photoelectron emission from Ne after XUV excitation may be accompanied by the promotion of loosely bound electrons to unoccupied orbitals (the so-called shake-up processes) [25]. The intense NIR field, which was utilized in our study to streak the photoelectrons can ionize these shake-up states, resulting in the creation of doubly charged ions and the emission of low energy electrons. The strong ATI signal from the interaction of Ne with the NIR field in the current measurements did not yet allow to study this process. Future experiments under optimized conditions and/or utilizing coincidence detection of ions and electrons may be able to discern electrons from the double ionization events from the (currently dominating) ATI background.

The fully angle-resolved experimental results on the relaxation dynamics of the inner shell $4 \mathrm{~d}$ vacancy in Xe can serve to test models for Auger decay in strong laser fields in the intermediate regime between the generation of sidebands and attosecond streaking. The obtained full three dimensional momentum distributions of the Auger electrons allowed us to monitor the Auger process simultaneously in both polarization directions. The results show reasonable agreement with the model calculations, which include the retrieved NIR vector potential from the streaking of photoemitted $4 \mathrm{~d}$ electrons as well as $\mathrm{N}_{4,5} \mathrm{OO}$ Auger decay times [24]. Although we have attempted an analysis of the temporal dependence of the angular distributions of the Auger electron emission, the limited signal-to-noise ratio in the current data did not allow distinguishing any significant temporal changes in the angular distribution. Future studies under improved conditions and potentially higher NIR intensities might help to elucidate the influence of the strong laser field on the Auger emission process, which was discussed in a recent publication for $\operatorname{Ar}[26]$.

Velocity-map imaging is shown to be a powerful tool for angle-resolved attosecond spectroscopy of photoemission and Auger processes in strong laser fields, the capabilities of which shall be further exploited in the future. This approach was not yet brought to its ultimate limit but holds substantial promises of elucidating strong field effects in multielectron decay correlations recorded over $4 \pi$ solid angles.

\section{Acknowledgements}

We acknowledge Ulf Kleineberg and Ferenc Krausz for their support and for making specialized equipment available to us. We are grateful for experimental support by Eleftherios Goulielmakis, Martin Schultze, and Michael Hofstetter and stimulating discussions of this work with Andrei Kazansky and Nikolay Kabachnik. We acknowledge support by the European network ATTOFEL, the Max Planck Society and the German Science Foundation (DFG) via the Emmy-Noether program, the International Collaboration in Chemistry program and the Cluster of Excellence: Munich Center for Advanced Photonics (MAP). The research of M.J.J.V. is part of the research program of the "Stichting voor Fundamenteel Onderzoek der Materie (FOM)", which is financially supported by the "Nederlandse organisatie voor Wetenschappelijk Onderzoek (NWO)". 
M.F.K. acknowledges support from the Chemical Sciences, Geosciences, and Biosciences Division, Office of Basic Energy Sciences, Office of Science, U.S. Department of Energy and the National Science Foundation under CHE-0822646.

\section{References}

[1] Zewail A H 2000 J. Phys. Chem. A 1045660

[2] Kling M F and Vrakking M J J 2008 Annu. Rev. Phys. Chem. 59463

[3] Krausz F and Ivanov M 2009 Rev. Mod. Phys. 81163

[4] Schartner K-H et al 1990 Phys. Scr. 41853

[5] Drescher M et al 2002 Nature 419803

[6] Uiberacker M et al 2007 Nature 446627

[7] Uphues T et al 2008 New. J. Phys. 10025009

[8] Smirnova O et al 2003 Phys. Rev. Lett. 91253001

[9] Kazansky A K et al 2009 J. Phys. B 42245601

[10] Kazansky A K and Kabachnik N M 2010 J. Phys. B 43035601

[11] Schultze M et al J. Elec. Spec. Rel. Phenom. In Press

[12] Ghafur O et al 2009 Rev. Sci. Instrum. 80033110

[13] Vrakking M J J 2001 Rev. Sci. Instr. 724084

[14] Itatani J et al 2002 Physical Review Letters 88173903

[15] Mauritsson J et al 2008 Phys. Rev. Lett. 100073003

[16] Kazansky A K and Kabachnik N M 2007 J. Phys. B 402163

[17] Kazansky A K and Kabachnik N M 2007 J. Phys. B 403413

[18] Gagnon J et al 2008 Appl. Phys. B 9225

[19] Wang H et al 2009 J. Phys. B 42134007

[20] Becker U et al 1989 Phys. Rev. A 393902

[21] Kämmerling B et al 1992 J. Phys. B 253621

[22] Carroll T X et al 2002 J. Elec. Spec. Rel. Phenom. 125127

[23] Werme L O et al 1972 Phys. Scripta 6141

[24] Penent F et al 2005 Phys. Rev. Lett. 95083002

[25] Holland D M P et al 1979 J. Phys. B 122465

[26] Ranitovic P et al 2011 Phys. Rev. Lett. 106053002 